\title{
The polarization-optical analysis of mechanics crack problems in rubber
}

\author{
G. Albaut1, M. Akhmetzyanov ${ }^{2}$ and N. Kharinova1 ${ }^{1}$ \\ ${ }^{1}$ Novosibirsk State University of Architecture and Civil Engineering, Russia \\ ${ }^{2}$ Novosibirsk state university of communication means, Civil Mechanics Department, Russia
}

At present time soft pneumatic environments, a various sort find rubber membranes, rubber products, shock-absorbers, etc have wide application in construction and a facilities recently. They work at the large elastic strains. The problem of definition stress-strain conditions at concentrators, i.e. in zones of prefraction and destruction of elements are especially difficult in conditions of the large nonlinearity.

Calculations of products from rubber are made by methods of the elasticity nonlinear theory [1]. The majority of works in this area have no full and precise theoretical decisions. They were obtained only for some most simple problems, for example, at homogeneous strains. Numerical methods for research of concentration problems at the large strains were developed insufficiently for the present.

In this work the mechanism of disclosing of internal cracks and cuts in rubber strips was studying by the experimental method of nonlinear photoelasticity [2]. Maximum strains were about $150 \%$ of relative lengthenings near concentrations.

As a result by polarization-optical experiment were obtained interference picture fringe patterns. The fringes order $\mathbf{n}$ at them represent in fact a field of a difference of main efforts $\lambda_{3}\left(\sigma_{1}-\sigma_{2}\right)$.

Tensioning of a strip with an internal crack. Rubber strips from polyurethane SKU-6 with the size of cross section $40 \times 1,5 \mathrm{~mm}$ were axial tensioned. The loading scheme was submitted on fig. 1. A crack in length $12 \mathrm{~mm}$ made in the middle of a model by sharp punch. The axial tensioning of strips with cracks was made at 15-20 stages with registration interference picture fringe patterns on each of them. Interference picture fringe patterns were made in a dark field polariscope at a various loading level of a model, fig. 1.

The analysis of disclosing of a crack at a tensioning of a model was submitted on fig. 1 from the right. During straining the crack was gradually opened, transforming to an ellipse, its sharp ends were became blunt. Also it was visible, that with increasing a vertical axis of a crack there was a reduction of its length.
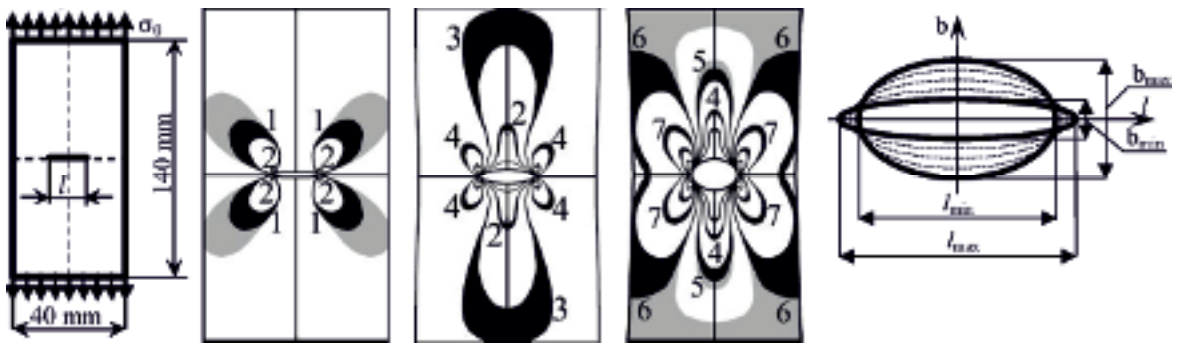

Fig. 1. Scheme of loading, interference picture fringe patterns and disclosing of a crack at a tensioning of a rubber strip with a horizontal crack 
Tensioning of strips with side cuts. Strips from rubber in width of $40 \mathrm{~mm}$ and thickness $1,5 \mathrm{Mm}$ with symmetric side cuts which were cut by a sharp scalpel were tensioned. The loading scheme of model was resulted on fig. 2. Depth of cuts made $l=10 \mathrm{~mm}$. The axial tensioning of strips with cuts was made by steps in some stages. At the initial tensioning cuts were closed.

Photos of interference picture fringe patterns at different steps models loading in a light field polariscope were made by of polarization-optical experiment. Fragments of some from them for a various level loading were resulted on fig. 2 .

The analysis of disclosing of side cuts at a tensioning of a model was submitted on fig. 2. It was visible, that at increase in loading there was a disclosing cuts and were became blunt their tops. Cuts, being gradually opened, got the form close to a parabola at an axial tensioning of models. It was distinctly visible in photos, that points on a contour of cuts in which there were maximal planimetric stresses were shifted from top of a cut (the concentrator) in both sides along a free contour. I.e. at top of a crack it was not observed not only indefinitely large stresses, but even their maximal values.
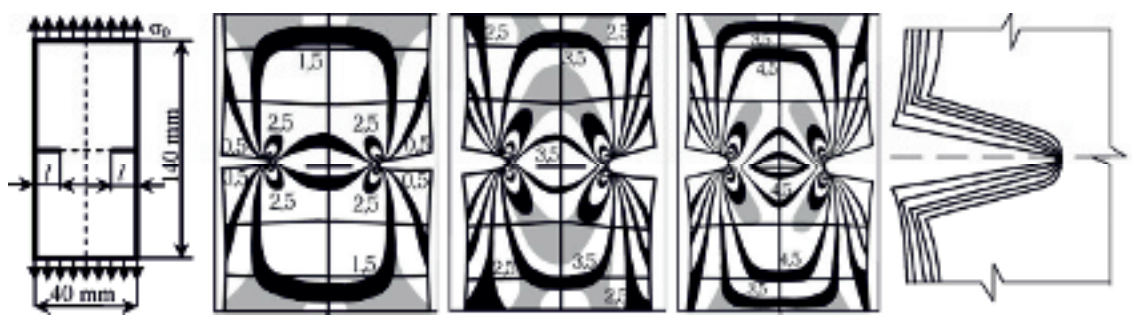

Fig. 2. Scheme of loading, interference picture fringe patterns and disclosing of side cuts at a tensioning of a rubber strip

\section{The conclusion}

The mechanism of $\mathrm{c}$ racks disclosing was experimentally established: to growth of deformations were became blunt sharp tops of crack and side cuts; crack transformed to ellipses, and side cuts had the form of a parabola.

Fields of running tangents of efforts were obtained at a tensioning of strips with central crack and side cuts (a interference picture fringe patterns).

Features in distribution of stresses were marked in zones of the preprefractions which were not conterminous to known theoretical decisions. So experimentally made points with the maximal values of stresses were shifted from tops of cracks - cuts in both sides along a contour of a cut and deep into a sample, fig. 1, 2 .

\section{References}

1. Chernykh K .F. Nonlinear elasticity (Theory and applications)/ Chernykh K.F.// SPb: publ. "Solo", 2004. $-420 \mathrm{p}$.

2. Albaut G. Photoelasticity study of stress-strain condition near cracks in rubber materials/ Albaut G., Kharinova N .// In Proceedings of 21st Symposium on experimental mechanics of solids. Jachranca, Poland. - 2004. - P.129-134. 